\title{
Quantum-geometry-induced intrinsic optical anomaly in multiorbital superconductors
}

\author{
Weipeng Chen $\odot$ and Wen Huang $\odot^{*}$ \\ Shenzhen Institute for Quantum Science and Engineering, Southern University of Science \\ and Technology, Shenzhen 518055, Guangdong, China; \\ International Quantum Academy (SIQA), and Shenzhen Branch, Hefei National Laboratory, Futian District, Shenzhen, China \\ and Guangdong Provincial Key Laboratory of Quantum Science and Engineering, Southern University of Science \\ and Technology, Shenzhen 518055, Guangdong, China
}

(Received 22 June 2021; accepted 20 October 2021; published 5 November 2021)

\begin{abstract}
Bloch electrons in multiorbital systems carry nontrivial quantum geometric information characteristic of their orbital composition as a function of their wave vector. When such electrons form Cooper pairs, the resultant superconducting state naturally inherits aspects of the quantum geometry. In this paper, we study how this geometric character is revealed in the intrinsic optical response of the superconducting state. In particular, due to the superconducting gap opening, interband optical transitions involving states around the Fermi level are forbidden. This generally leads to an anomalous suppression of the optical conductivity at frequencies matching the band separation - which could be significantly higher than the superconducting gap energy. We discuss how the predicted anomaly may have already emerged in two earlier measurements on an iron-based superconductor. When interband Cooper pairing is present, intraband optical transitions may be allowed and finite conductivity emerges at low frequencies right above the gap edge. These conductivities depend crucially on the off-diagonal elements of the velocity matrix in the band representation, i.e., the interband velocity-which is related to the non-Abelian Berry connection of the Bloch states.
\end{abstract}

DOI: 10.1103/PhysRevResearch.3.L042018

\section{INTRODUCTION}

In a multiorbital system, Bloch electrons on the individual energy bands are generally not featureless particles. They exhibit nontrivial internal structure, in the sense that they possess quantum geometric information associated with their varying orbital composition as a function of the wave vector. This property manifests in the quantum transport: the electrical currents carried by the Bloch electrons in such multiorbital setting, having their origin in the physical motion of the underlying orbitals, are not determined solely by the group velocities of the individual Bloch states.

Let us illustrate the idea using the example of a general two-orbital model. We consider the two orbital degrees of freedom stemming from either two different electron orbitals residing on a monatomic lattice, or orbitals of the same symmetry each occupying one sublattice of a bipartite lattice. The kinetic Hamiltonian is given by $H=\sum_{\mathbf{k}} \psi_{\mathbf{k}}^{\dagger} \hat{H}_{\mathbf{k}}^{\text {orb }} \psi_{\mathbf{k}}$, where $\psi_{\mathbf{k}}^{\dagger}=\left(a_{\mathbf{k}}^{\dagger}, b_{\mathbf{k}}^{\dagger}\right)$ in which $a_{\mathbf{k}}^{\dagger}$ and $b_{\mathbf{k}}^{\dagger}$ stand for the creation operators of the respective $a$ and $b$ orbitals, and $\hat{H}_{\mathbf{k}}^{\text {orb }}$ generally takes the form

$$
\hat{H}_{\mathbf{k}}^{\text {orb }}=\left(\begin{array}{cc}
\xi_{a, \mathbf{k}} & \lambda_{\mathbf{k}} \\
\lambda_{\mathbf{k}}^{*} & \xi_{b, \mathbf{k}}
\end{array}\right)
$$

\footnotetext{
*huangw3@sustech.edu.cn

Published by the American Physical Society under the terms of the Creative Commons Attribution 4.0 International license. Further distribution of this work must maintain attribution to the author(s) and the published article's title, journal citation, and DOI.
}

Here, $\xi_{a(b), \mathbf{k}}$ represent the dispersion relation of the two orbitals in the unhybridized limit and $\lambda_{\mathbf{k}}$ describes the orbital mixing. For brevity, we have ignored spin-orbit coupling (SOC) for now and suppressed the spin indices. The forms of $\lambda_{\mathbf{k}}$ and $\xi_{\mathbf{k}}=\xi_{a, \mathbf{k}}-\xi_{b, \mathbf{k}}$ are essential for characterizing the quantum geometry $(\mathrm{QG})$ of the resultant Bloch electrons. Since electrical current is generated by the hopping of the orbitals on the lattice, it is connected to the Hamiltonian (1) via the relation $J_{\mu, \mathbf{k}}=e \psi_{\mathbf{k}}^{\dagger} \hat{V}_{\mu, \mathbf{k}}^{\text {orb }} \psi_{\mathbf{k}}$, where $\hat{V}_{\mu, \mathbf{k}}^{\text {orb }}=\partial_{k_{\mu}} \hat{H}_{\mathbf{k}}^{\text {orb }}$ is the velocity operator. Formally, the operator is obtained from the standard Peierls substitution by changing $\mathbf{k}$ to $\mathbf{k}-e \mathbf{A}$ in the kinetic Hamiltonian and then taking $\partial \hat{H}_{\mathbf{k}-e \mathbf{A}}^{\text {orb }} /\left.\partial \mathbf{A}\right|_{\mathbf{A} \rightarrow 0}$. This operator can be rewritten in the (Bloch) band basis through a unitary transformation. We write $J_{\mu, \mathbf{k}}=e \phi_{\mathbf{k}}^{\dagger} \hat{V}_{\mu, \mathbf{k}} \phi_{\mathbf{k}}$, where $\phi_{\mathbf{k}}^{\dagger}=\left(c_{1, \mathbf{k}}^{\dagger}, c_{2, \mathbf{k}}^{\dagger}\right)$ with 1,2 denoting the band indices, and

$$
\hat{V}_{\mu, \mathbf{k}}=\hat{\mathcal{V}}_{\mathbf{k}}^{-1} \partial_{k_{\mu}} \hat{H}_{\mathbf{k}}^{\mathrm{orb}} \hat{\mathcal{V}}_{\mathbf{k}}=\left(\begin{array}{cc}
V_{\mu, \mathbf{k}}^{11} & V_{\mu, \mathbf{k}}^{12} \\
V_{\mu, \mathbf{k}}^{21} & V_{\mu, \mathbf{k}}^{22}
\end{array}\right),
$$

where $\hat{\mathcal{V}}_{\mathbf{k}}$ is a unitary matrix that diagonalizes $\hat{H}_{\mathbf{k}}^{\text {orb }}$. The diagonal elements of (2), i.e., the intraband velocities $V_{\mu, \mathbf{k}}^{n n}$, can be shown to simply equal the band group velocities. The interband velocity, on the other hand, is given by

$$
\begin{aligned}
V_{\mu, \mathbf{k}}^{m n} & =\left\langle m, \mathbf{k}\left|\partial_{k_{\mu}} \hat{H}_{\mathbf{k}}^{\mathrm{orb}}\right| n, \mathbf{k}\right\rangle \\
& =\left(\epsilon_{m, \mathbf{k}}-\epsilon_{n, \mathbf{k}}\right)\left\langle m, \mathbf{k}\left|\partial_{k_{\mu}}\right| n, \mathbf{k}\right\rangle(m \neq n),
\end{aligned}
$$

where $\epsilon_{m, \mathbf{k}}$ is the energy dispersion of the $m$ th band with eigenvector $|m, \mathbf{k}\rangle$. The object $i\left\langle m, \mathbf{k}\left|\partial_{k_{\mu}}\right| n, \mathbf{k}\right\rangle$ is known as the non-Abelian Berry connection and is involved in the definition of the quantum geometric tensor [1-6]. It is finite provided the orbital composition of the Bloch states, i.e., the rela- 
tive amplitude and/or relative phase of the orbitals involved, vary continuously in momentum space. By inspection, this is equivalent to stating that (1) the orbital mixing $\lambda_{\mathbf{k}}$ is finite and (2) at least one of $\xi_{\mathbf{k}}$ and $\lambda_{\mathbf{k}}$ is a varying function of $\mathbf{k}$. The interband velocity showcases a profound consequence of the QG, because it depicts how electrons from distinct energy bands still "talk" to each other as far as charge transport is concerned.

In a weak-coupling superconducting state, electrons on the Fermi level most actively participate in Cooper pairing. Without considering interband pairing, the fermionic excitation spectra of the two bands are decoupled. However, by the same reasoning stated above, the Bogoliubov quasiparticles of one band shall still inherently connect to the unpaired electrons of the other via the QG. It is thus natural to expect this quantum connection to reveal itself in the superconducting electromagnetic response. There were previous studies along similar direction about the band geometric effect on the superfluid stiffness in flat-band systems [2,7,8], and applications to theories of the twisted bilayer graphene followed recently [9-13]. More of its unusual aspects await investigation. In this paper, we demonstrate how the geometric character of the superconducting electrons influences the intrinsic optical conductivity.

\section{FORMULATION}

In what follows, we will consistently formulate our theory in the band representation, for both normal and superconducting states. In this basis, the eigenvectors of the individual bands acquire the simple forms $(1,0)^{T}$ and $(0,1)^{T}$, for which we continue to use the designation $|m(n), \mathbf{k}\rangle$. Let us first consider the normal-state response. Within linear-response theory, the conductivity is given by the following currentcurrent correlator:

$$
\sigma_{n}(\omega)=\frac{T}{\omega} \sum_{\mathbf{k}, \omega_{n}} \operatorname{Tr}\left[\hat{V}_{x, \mathbf{k}} \hat{G}_{\mathbf{k}}\left(\omega_{n}\right) \hat{V}_{x, \mathbf{k}} \hat{G}_{\mathbf{k}}\left(\omega_{n}+\omega\right)\right] .
$$

We shall focus on the real part of $\sigma(\omega)$. Expressing the Greens' function in the spectral representation, one obtains, for the real part that we shall focus on,

$$
\operatorname{Re}\left[\sigma_{n}(\omega)\right]=\sum_{m, n, \mathbf{k}}\left|\left\langle m, \mathbf{k}\left|\hat{V}_{x, \mathbf{k}}\right| n, \mathbf{k}\right\rangle\right|^{2} F\left(\omega ; \epsilon_{m, \mathbf{k}}, \epsilon_{n, \mathbf{k}}\right) .
$$

We have adopted a shorthand expression $F\left(\omega ; x_{1}, x_{2}\right)=$ $\left[f\left(x_{1}\right)-f\left(x_{2}\right)\right] \delta\left(\omega+x_{1}-x_{2}\right) / \omega$, where $f(x)$ stands for the Fermi distribution function. At zero temperature, it has finite value only at wave vectors satisfying $\epsilon_{m, \mathbf{k}}<0<\epsilon_{n, \mathbf{k}}$ and is therefore cut off in momentum space by the Fermi surface(s). The corresponding "cutoff" frequencies $\omega_{\mathrm{F}}$ (Fig. 1), which measure the band separation at the Fermi wave vectors, are important quantities to keep in mind.

Obviously, only momentum-conserving interband transitions (i.e., $m \neq n$ ) contribute, with $\left\langle 2, \mathbf{k}\left|\hat{V}_{x, \mathbf{k}}\right| 1, \mathbf{k}\right\rangle=V_{x, \mathbf{k}}^{21}$. Hence the interband velocity fully characterizes the geometric footprints in the intrinsic conductivity. Note that simply inserting the identity operator $\hat{\mathcal{V}}_{\mathbf{k}}^{-1} \hat{\mathcal{V}}_{\mathbf{k}}$ in between the $\hat{V}_{x}$ 's and $\hat{G}$ 's in the first line of (4) restores the expression to the orbital basis formulation, and the same result will follow.
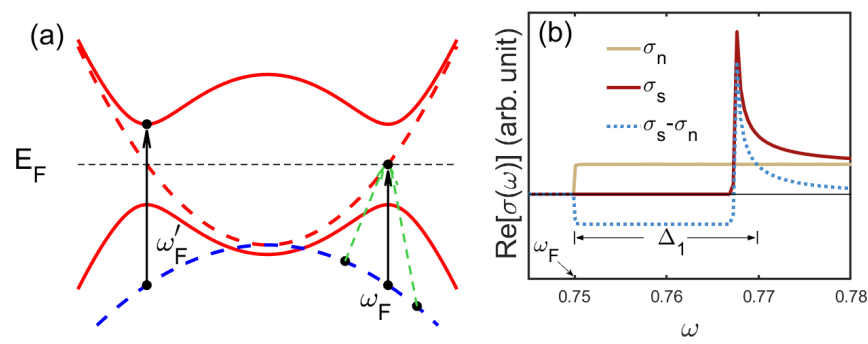

FIG. 1. (a) Schematic interband optical transitions (arrows) in a $d_{x z}-d_{y z}$ model with only one band crossing the Fermi level. Dashed curves sketch the normal-state band structure, and the red solid curves the Bogoliubov quasiparticle spectra of the superconducting band. The black arrows denote transitions that contribute to the intrinsic optical transitions in the normal (dotted) and superconducting (solid) states, while the green dotted arrows exemplify momentum-nonconserving transitions induced by disorder scattering in normal state. (b) The real part of the optical conductivity of normal and superconducting phases near the cutoff frequency $\omega_{F} \approx 0.75$. The dotted curve shows their difference, which exhibits a dip between $\omega_{F}$ and roughly $\omega_{F}+\Delta_{1}$. In the calculation, we took $\xi_{a(b), \mathbf{k}}=\frac{k_{x}^{2}}{2 m_{\|(\perp)}}+\frac{k_{y}^{2}}{2 m_{\perp(\|)}}-\mu$ and $\lambda_{\mathbf{k}}=\lambda k_{x} k_{y}$, with $\left(m_{\|}, m_{\perp}, \mu, \lambda\right)=$ $(1,-2,0.5,-0.75)$. Superconducting pairing is assumed to take place only on the band that crosses the Fermi level, with pairing gap $\Delta_{1}=0.02$.

Turning to the superconducting state, we consider for simplicity a scenario where only one of the bands (say, band 1) crosses the Fermi energy and superconducts with a simple constant $s$-wave gap, while the other sits below the Fermi energy [Fig. 1(a)]. The general Bogoliubov-de Gennes (BdG) Hamiltonian reads

$$
H_{\mathrm{BdG}}=\sum_{\mathbf{k}, n, s} \epsilon_{n, \mathbf{k}} c_{n, \mathbf{k} s}^{\dagger} c_{n, \mathbf{k} s}+\sum_{\mathbf{k}} \Delta_{1, \mathbf{k}} c_{1, \overline{\mathbf{k}} \uparrow} c_{1, \mathbf{k} \downarrow}+\text { H.c. }
$$

In the sub-block associated with the Nambu basis $\left(c_{1, \mathbf{k} \uparrow}, c_{2, \mathbf{k} \uparrow}, c_{1, \overline{\mathbf{k}} \downarrow}^{\dagger}, c_{2, \overline{\mathbf{k}} \downarrow}^{\dagger}\right)^{T}$, where $\overline{\mathbf{k}}=-\mathbf{k}$, the velocity operator becomes

$$
\begin{aligned}
\tilde{V}_{x, \mathbf{k}} & =\left(\begin{array}{ll}
\hat{\mathcal{V}}_{\mathbf{k}}^{-1} \partial_{k_{x}} \hat{H}_{\mathbf{k}} \hat{\mathcal{V}}_{\mathbf{k}} & \hat{\mathcal{V}}_{\overline{\mathbf{k}}}^{*-1} \partial_{k_{x}} \hat{H}_{\mathbf{\mathbf { k }}}^{*} \hat{\mathcal{V}}_{\mathbf{\mathbf { k }}}^{*}
\end{array}\right) \\
& =\left(\begin{array}{ll}
\hat{V}_{x, \mathbf{k}} & -\hat{V}_{x, \overline{\mathbf{k}}}^{*}
\end{array}\right) .
\end{aligned}
$$

Generalizing (4) to the superconducting state, one arrives at an expression for $\operatorname{Re}\left[\sigma_{s}(w)\right]$ similar to (5), but with $\epsilon_{m(n), \mathbf{k}}$ and $|m(n), \mathbf{k}\rangle$ replaced respectively by the Bogoliubov quasiparticle dispersion and eigenvectors.

As in the normal state, virtual excitations that contribute to the conductivity involve interband processes. A representative transition is indicated by the solid black arrow in Fig. 1 between a negative-energy state associated with band 2, $|\overline{2}, \mathbf{k}\rangle_{s}=(0,1,0,0)^{T}$, and a positive-energy state associated with band $1,|1, \mathbf{k}\rangle_{s}=\left(\Delta_{1, \mathbf{k}}, 0, E_{1, \mathbf{k}}-\epsilon_{1, \mathbf{k}}, 0\right)^{T} / \mathcal{N}_{1, \mathbf{k}}$ where $E_{1, \mathbf{k}}=\left(\epsilon_{1, \mathbf{k}}^{2}+\Delta_{1, \mathbf{k}}^{2}\right)^{1 / 2}$ and $\mathcal{N}_{1, \mathbf{k}}=\sqrt{2 E_{1, \mathbf{k}}\left(E_{1, \mathbf{k}}-\epsilon_{1, \mathbf{k}}\right)}$ is a normalization factor. Note that we use the subscript " $s$ " to designate states in the superconducting state. One then obtains 

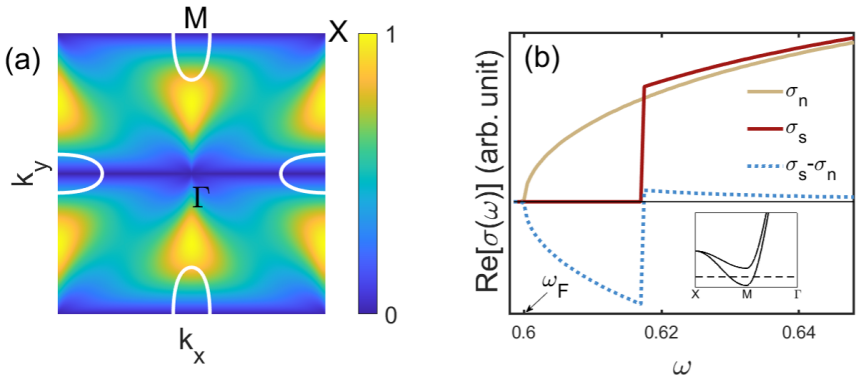

FIG. 2. (a) Fermi surface (white contour) and normalized magnitude of the interband velocity $\left|V_{x, \mathbf{k}}^{12}\right|$ in a square lattice $d_{x z}-d_{y z}$ model. (b) The real part of the optical conductivity in the normal and superconducting states, and their difference. The model has $\xi_{a(b), \mathbf{k}}=$ $-2 t_{\|(\perp)} \cos k_{x}-2 t_{\perp(\|)} \cos k_{y}-4 t^{\prime} \cos k_{x} \cos k_{y}-\mu \quad$ and $\quad \lambda_{a b, \mathbf{k}}=$ $\lambda \sin k_{x} \sin k_{y}$, where $\left(t_{\|}, t_{\perp}, t^{\prime}, \lambda, \mu\right)=(1,0.8,-0.6,0.4,-2.4)$. Inset shows the band structure near the $M$ point. Only one band crosses Fermi level with a pairing gap $\Delta_{1}=0.02$.

a transition matrix element

$$
{ }_{s}\left\langle\overline{2}, \mathbf{k}\left|\widetilde{V}_{x, \mathbf{k}}\right| 1, \mathbf{k}\right\rangle_{s}=\frac{\Delta_{1, \mathbf{k}}}{\mathcal{N}_{1, \mathbf{k}}} V_{x, \mathbf{k}}^{21} .
$$

Another process involving their counterpart particle-holesymmetric states $|2, \overline{\mathbf{k}}\rangle_{s}$ and $|\overline{1}, \overline{\mathbf{k}}\rangle_{s}$ leads to the same expression. Put together, and with a proper account of the double-counting in Nambu representation, the full expression for the real part of conductivity becomes

$$
\operatorname{Re}\left[\sigma_{s}(w)\right]=\sum_{\mathbf{k}}\left|\frac{\Delta_{1, \mathbf{k}}}{\mathcal{N}_{1, \mathbf{k}}} V_{x, \mathbf{k}}^{21}\right|^{2} F\left(\omega ;-E_{2, \mathbf{k}}, E_{1, \mathbf{k}}\right),
$$

where $E_{2, \mathbf{k}}=\left|\epsilon_{2, \mathbf{k}}\right|$ for an unpaired band 2. Due to the superconducting gap opening in band 1 , transitions involving states in the immediacy of the Fermi surface are forbidden. As a consequence, the conductivity in the superconducting state shall show an anomalous suppression within a width of $\Delta_{1}$ around the cutoff frequencies defined above. On the other hand, a pileup of density of states at the continuum edge shall give rise to a peak right above $\omega_{F}^{\prime} \approx \omega_{F}+\Delta_{1}$. This is confirmed in Fig. 1 using a continuum two-orbital model with $d_{x z}$ and $d_{y z}$ orbitals.

In lattice models with band anisotropy, the peak structure could be smeared out. We now generalize the above analysis to a square lattice model. In a representative calculation shown in Fig. 2, our model produces two Fermi pockets at the $\mathbf{M}$ points of the Brillouin zone (BZ) - reminiscent of the scenario in some iron-based superconductors [14]. Figure 2(a) depicts the momentum-space distribution of the absolute value of the $x$ component of the interband velocity. Notably, unlike the intraband velocities, it does not vanish at $k_{x}=0, \pi$. As shown in Fig. 2(b), $\operatorname{Re}\left[\sigma_{s}(\omega)-\sigma_{n}(\omega)\right]$ no longer exhibits the peak structure, whereas the dip above $\omega_{F}$ persists.

Besides anisotropy, real materials always contain finite amount of disorder scatterings which enable momentumnonconserving interband transitions. As a consequence, the gap opening prohibits not only the "direct" optical transitions at $\omega_{F}$ but also some "indirect" transitions as exemplified by the green dashed arrows in Fig. 1(a). Hence the anomalous suppression is not necessarily restricted to a narrow width of
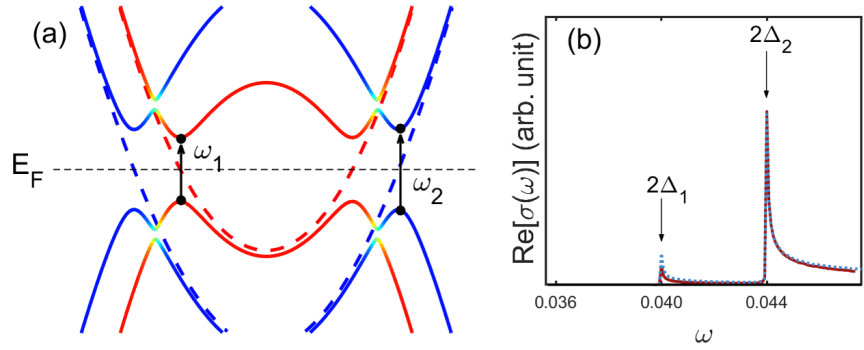

FIG. 3. (a) Schematic intraband optical transitions (black arrows) induced by interband Cooper pairing. The dashed and solid curves depict the energy dispersion in the normal and superconducting states of a continuum $d_{x z}-d_{y z}$ model with finite SOC. (b) Real part of the low-frequency optical conductivity induced by interband pairing. The solid (red) and dotted (blue) curves show results obtained from the numerical calculation and the perturbative expansion, respectively. The continuum Hamiltonian with SOC is given in the Supplemental Material [17] and we took $\left(m_{\|}, m_{\perp}, \mu, \lambda, \eta\right)=$ $(1,0.2,0.8,0.5,0.3)$. The pairing amplitudes are $\left(\Delta_{1}, \Delta_{2}, \Delta_{12}\right)=$ $(0.02,0.022,0.005)$ and the constant $\gamma=1$.

$\Delta_{1}$ above $\omega_{F}$, but could potentially take place at all frequencies associated with indirect band separations, which amount to a frequency span as wide as the bandwidth of the unpaired band. Interestingly, one such optical anomaly was indeed detected in an earlier measurement on the iron-based superconducting compound $\mathrm{Ba}_{0.68} \mathrm{~K}_{0.32} \mathrm{Fe}_{2} \mathrm{As}_{2}$ [15]. There, the superconductivity-induced conductivity suppression centers around $\omega=2.5 \mathrm{eV}$ and spans a broad frequency range of $1 \mathrm{eV}$. The effect was ascribed to interband transitions involving the strongly hybridized Fe- $d$ and As- $p$ orbitals.

A more pronounced anomaly was reported in the same material at lower frequencies above the gap edge, and was attributed to spin-fluctuation-assisted scattering effects among the multiple bands dominated by the Fe- $d$ orbitals near the Fermi energy [16]. However, as we describe in detail in the Supplemental Material [17], our theory is also able to produce a qualitatively similar anomalous suppression, using a model that captures some essential features of the band and gap structure of this material around the $\Gamma$ point [18].

\section{INTERBAND-PAIRING-INDUCED CONDUCTIVITY}

It must be stressed that, in the absence of interband Cooper pairing, low-energy intraband transitions [Fig. 3(a)] are absent in the clean limit. This explains the vanishing of $\operatorname{Re}\left[\sigma_{s}(\omega)\right]$ at low frequencies. It is also reminiscent of the absence of longitudinal excitations in single-band superconductors under the perturbation of a weak uniform magnetic field [19], i.e., $H^{\prime}=\int d \mathbf{r} e \hat{\boldsymbol{V}} \cdot \mathbf{A}$, which underlies the Meissner effect therein. One way to see the connection is by recognizing, in single-band systems, the vanishing of ${ }_{s}\left\langle\bar{m}, \mathbf{k}\left|\tilde{V}_{\mu, \mathbf{k}}\right| m, \mathbf{k}\right\rangle_{s}$, where $\tilde{V}_{\mu, \mathbf{k}}$ is proportional to an identity matrix in this case.

One natural question is how interband pairing affects the low-frequency electromagnetic response. As we focus on translation invariant models, we only consider interband Cooper pairs formed between electrons of opposite wave vectors. Such interband pairing is customarily ignored in much of the literature, however, there exists no symmetry constraint 
to forbid it in realistic multiband superconductors. In fact, it is particularly relevant for systems where the band separation is small, and more so for superconducting pairings driven by electron-electron correlations-where the Coulomb-derived effective attractive interactions are not necessarily confined to a thin layer of phase space around the Fermi surface [20].

We illustrate the idea in the continuum limit of the squarelattice $d_{x z}-d_{y z}$ model. For generality purpose and to avoid ambiguity at $\mathbf{k}=0$ due to band degeneracy, we add a finite SOC. We further assume that both bands cross the Fermi level and superconduct, and that they are proximate in energy, making it more reasonable to speak about sizable interband pairing in the system.

Around $\mathbf{k}=0$, the two bands are classified according to their total angular-momentum eigenvalues $J=L+S$, designated as follows:

$$
\begin{array}{r}
\text { Band 1: }\left|j_{z}=\frac{3}{2}\right\rangle=\left|d_{x z}+i d_{y z}\right\rangle \otimes|\uparrow\rangle, \\
\left|\begin{array}{l}
\left.j_{z}=-\frac{3}{2}\right\rangle \\
\mid
\end{array}\right|=\left|d_{x z}-i d_{y z}\right\rangle \otimes|\downarrow\rangle, \\
\text { Band 2: } \quad\left|j_{z}=-\frac{1}{2}\right\rangle=\left|d_{x z}-i d_{y z}\right\rangle \otimes|\uparrow\rangle, \\
\left|j_{z}=\frac{1}{2}\right\rangle=\left|d_{x z}+i d_{y z}\right\rangle \otimes|\downarrow\rangle .
\end{array}
$$

The symmetry properties of these states directly affect the forms of the interband pairings, which requires a special attention because they in general do not share the same basis functions as that of the intraband pairing [21]. This can be understood by noting that, when acting on an interband pairing $\Delta_{12, \mathbf{k}} c_{1, \overline{\mathbf{k}}} c_{2, \mathbf{k}}$, any symmetry operation must simultaneously transform $\Delta_{12, \mathbf{k}}$, as well as the wave functions of the two constituent electrons which exhibit distinct symmetries.

We take as an example a superconducting state with $A_{1 g}$ symmetry in the $D_{4 h}$ point group, where the intraband pairings on the two bands acquire usual $s$-wave forms. As is analyzed more systematically in the Supplemental Material [17,22], the interband pairing develops only between $j_{z}=\frac{3}{2}$ and $j_{z}=\frac{1}{2}$ states and between $j_{z}=-\frac{3}{2}$ and $j_{z}=-\frac{1}{2}$ states, and it shall acquire a general form of $\left(k_{x}^{2}-k_{y}^{2}\right)+i \gamma k_{x} k_{y}$, where $\gamma$ is an inessential real constant that depends on microscopic details. Such a pairing combines with the symmetries of the constituent electrons to yield an overall $A_{1 g}$ symmetry. Consider, for instance, a $C_{4}$ rotation. It changes $\Delta_{12, \mathbf{k}}$ to $-\Delta_{12, \mathbf{k}}, \mid j_{z}=$ $\left.\frac{3}{2}\right\rangle$ to $e^{i \frac{3 \pi}{4}}\left|j_{z}=\frac{3}{2}\right\rangle$, and $\left|j_{z}=\frac{1}{2}\right\rangle$ to $e^{i \frac{\pi}{4}}\left|j_{z}=\frac{1}{2}\right\rangle$. Hence, the interband pairing is invariant under $C_{4}$, and the same holds under other symmetry operations. Treated as a perturbation to the original $\mathrm{BdG}$ Hamiltonian, the interband pairing can be written, in the sub-basis $\left(c_{\frac{3}{2}, \mathbf{k}}, c_{-\frac{1}{2}, \mathbf{k}}, c_{-\frac{3}{2}, \overline{\mathbf{k}}}^{\dagger}, c_{\frac{1}{2}, \overline{\mathbf{k}}}^{\dagger}\right)^{T}$, as [17]

$$
\hat{H}_{\delta, \mathbf{k}}=\left(\begin{array}{cccc} 
& & & \Delta_{12, \mathbf{k}} \\
& \Delta_{21, \mathbf{k}}^{*} & & \\
\Delta_{12, \mathbf{k}}^{*} & & &
\end{array}\right),
$$

where $\Delta_{12, \mathbf{k}}=\Delta_{21, \mathbf{k}}^{*}=\Delta_{12}\left[\left(k_{x}^{2}-k_{y}^{2}\right)+i \gamma k_{x} k_{y}\right]$. By secondorder perturbation theory, the intraband transition matrix element follows as

$$
\begin{aligned}
& { }_{s}\left\langle\bar{m}^{\prime}, \mathbf{k}\left|\tilde{V}_{\mu, \mathbf{k}}\right| m^{\prime}, \mathbf{k}\right\rangle_{s} \\
= & \left(\alpha_{I, \mathbf{k}}-\alpha_{I I, \mathbf{k}}\right)\left(V_{\mu, \mathbf{k}}^{n m} u_{m} v_{n}-V_{\mu, \mathbf{k}}^{m n} v_{m} u_{n}\right) \\
+ & \left(\beta_{I, \mathbf{k}}-\beta_{I I, \mathbf{k}}\right)\left(V_{\mu, \mathbf{k}}^{n m} u_{m} u_{n}^{*}+V_{\mu, \mathbf{k}}^{m n} v_{m} v_{n}^{*}\right)(m \neq n) .
\end{aligned}
$$

Here, $\left|m^{\prime}, \mathbf{k}\right\rangle_{s}$ and $\left|\bar{m}^{\prime}, \mathbf{k}\right\rangle_{s}$ with $m=1,2$ denote the respective perturbed particle- and hole-like quasiparticle states associated with the corresponding bands, $\left(u_{m}, v_{m}\right)=\left(\Delta_{m, \mathbf{k}}, E_{m, \mathbf{k}}-\right.$ $\left.\epsilon_{m, \mathbf{k}}\right) / \mathcal{N}_{m, \mathbf{k}}$, and the remaining coefficients are linearly related to the interband pairing as follows:

$$
\begin{aligned}
\alpha_{I, \mathbf{k}} & =\frac{\Delta_{m n, \mathbf{k}}^{*} u_{m} v_{n}^{*}+\Delta_{n m, \mathbf{k}} v_{m} u_{n}^{*}}{E_{n, \mathbf{k}}-E_{m, \mathbf{k}}}, \\
\alpha_{I I, \mathbf{k}} & =\frac{\Delta_{n m, \mathbf{k}}^{*} u_{m} v_{n}^{*}+\Delta_{m n, \mathbf{k}} v_{m} u_{n}^{*}}{E_{n, \mathbf{k}}-E_{m, \mathbf{k}}}, \\
\beta_{I, \mathbf{k}} & =\frac{-\Delta_{m n, \mathbf{k}}^{*} u_{m} u_{n}+\Delta_{n m, \mathbf{k}} v_{m} v_{n}}{E_{n, \mathbf{k}}+E_{m, \mathbf{k}}}, \\
\beta_{I I, \mathbf{k}} & =\frac{-\Delta_{n m, \mathbf{k}}^{*} u_{m} u_{n}+\Delta_{m n, \mathbf{k}} v_{m} v_{n}}{E_{n, \mathbf{k}}+E_{m, \mathbf{k}}}
\end{aligned}
$$

The dependence on the interband velocity, and therefore on the QG, is evident in (11). Figure 3(b) presents the numerically evaluated low-frequency optical conductivity for a model where the intraband pairings on the two bands are comparable and the interband pairing amplitude is roughly four times smaller. Also drawn is the result obtained from the perturbative analysis (blue dotted), which shows excellent agreement. The two prominent peaks correspond to the gap edge of the two respective bands.

Ahn and Nagaosa [23] have also studied the low-frequency intrinsic conductivity of similar origin. Approached from a pure orbital-basis perspective, they identified certain symmetry criterion that could enable intraband transitions. We checked that our model falls in the category of class DIII in their effective Altland-Zirnbauer symmetry classification, where intraband excitation are allowed when there exists finite SOC. Our study contributes two significant advances: one is to pinpoint interband pairing as an essential ingredient for generating low-frequency intrinsic conductivity, and the other is to emphasize the critical role of the QG.

\section{CONCLUSION}

The quantum geometric character of the superconducting Bloch electrons in multiband systems has largely gone unnoticed until recently. In this paper, we demonstrate how it influences the intrinsic optical conductivity in a nontrivial fashion. We show that, as interband transitions involving quasiparticles around the Fermi level are forbidden due to superconducting gap opening, the optical conductivity shall generally exhibit an anomalous suppression at frequencies matching the band separation. The presence of interband Cooper pairing may further enable low-energy intraband optical transitions, thereby inducing low-frequency conductivity in the clean limit. These intrinsic contributions to the conductivity are closely related to the interband velocity, which, by itself, is a manifestation of the quantum geometry of the Bloch electrons. We expect our theory to be applicable to a 
broad spectrum of superconductors which exhibit multiband or multiorbital character, and we point out the possible connection to previously reported optical conductivity anomalies in an iron-based superconductor.

Note added. Recently, we became aware of a similar work by Ahn and Nagaosa [24], which also studied the anomalous changes in the optical spectral weight at high frequencies due to Bloch quantum geometry. The authors further pointed out the potential relevance to the long-standing puzzle of spectral weight transfer reported in underdoped and optimally doped cuprate superconductors (see, e.g., Ref. [25]).

\section{ACKNOWLEDGMENTS}

We acknowledge helpful discussions with Junfeng Dai, Jiawei Mei, Nanlin Wang, and Zhongbo Yan. We are also indebted to Alexander Boris for drawing our attention to Ref. [16] and for his comments. This work is supported by NSFC under Grant No. 11904155 and the Guangdong Provincial Key Laboratory under Grant No. 2019B121203002. Computing resources are provided by the Center for Computational Science and Engineering at Southern University of Science and Technology.
[1] J. Provost and G. Vallee, Riemannian structure on manifolds of quantum states, Commun. Math. Phys. 76, 289 (1980).

[2] L. Liang, T. I. Vanhala, S. Peotta, T. Siro, A. Harju, and P. Törmä, Band geometry, Berry curvature, and superfluid weight, Phys. Rev. B 95, 024515 (2017).

[3] M. Iskin, Geometric mass acquisition via a quantum metric: An effective-band-mass theorem for the helicity bands, Phys. Rev. A 99, 053603 (2019).

[4] Z. Li, S. Zhang, T. Tohyama, X. Song, Y. Gu, T. Iitaka, H. Su, and H. Zeng, Optical detection of quantum geometric tensor in intrinsic semiconductors, Sci. China Phys. Mech. Astron. 64, 107211 (2021).

[5] G. E. Topp, C. J. Eckhardt, D. M. Kennes, M. A. Sentef, and P. Törmä, Light-matter coupling and quantum geometry in more materials, Phys. Rev. B 104, 064306 (2021).

[6] J. Ahn, G.-Y. Guo, N. Nagaosa, and A. Vishwanath, Riemannian geometry of resonant optical responses, arXiv:2103.01241.

[7] S. Peotta and P. Törmä, Superfluidity in topologically nontrivial flatbands, Nat. Commun. 6, 8944 (2015).

[8] A. Julku, S. Peotta, T. I. Vanhala, D. H. Kim, and P. Törmä, Geometric Origin of Superfluidity in the Lieb-Lattice Flat Band, Phys. Rev. Lett. 117, 045303 (2016).

[9] X. Hu, T. Hyart, D. I. Pikulin, and E. Rossi, Geometric and Conventional Contribution to the Superfluid Weight in Twisted Bilayer Graphene, Phys. Rev. Lett. 123, 237002 (2019).

[10] A. Julku, T. J. Peltonen, L. Liang, T. T. Heikkilä, and P. Törmä, Superfluid weight and Berezinskii-Kosterlitz-Thouless transition temperature of twisted bilayer graphene, Phys. Rev. B 101, 060505(R) (2020).

[11] F. Xie, Z. Song, B. Lian, and B. A. Bernevig, TopologyBounded Superfluid Weight in Twisted Bilayer Graphene, Phys. Rev. Lett. 124, 167002 (2020).

[12] T. Hazra, N. Verma, and M. Randeria, Bounds on the Superconducting Transition Temperature: Applications to Twisted Bilayer Graphene and Cold Atoms, Phys. Rev. X 9, 031049 (2019).

[13] N. Verma, T. Hazra, and M. Randeria, Optical spectral weight, phase stiffness and $T_{c}$ bounds for trivial and topological flat band superconductors, Proc. Natl. Acad. Sci. 118, e2106744118 (2021).
[14] In reality, their band structure takes more than two orbitals to model.

[15] A. Charnukha, P. Popovich, Y. Matiks, D. Sun, C. Lin, A. Yaresko, B. Keimer, and A. Boris, Superconductivity-induced optical anomaly in an iron arsenide, Nat. Commun. 2, 219 (2011).

[16] A. Charnukha, O. V. Dolgov, A. A. Golubov, Y. Matiks, D. L. Sun, C. T. Lin, B. Keimer, and A. V. Boris, Eliashberg approach to infrared anomalies induced by the superconducting state of $\mathrm{Ba}_{0.68} \mathrm{~K}_{0.32} \mathrm{Fe}_{2} \mathrm{As}_{2}$ single crystals, Phys. Rev. B 84, 174511 (2011).

[17] See Supplemental Material at http://link.aps.org/supplemental/ 10.1103/PhysRevResearch.3.L042018 for a calculation of lower-frequency conductivity in a model of $\mathrm{Ba}_{0.68} \mathrm{~K}_{0.32} \mathrm{Fe}_{2} \mathrm{As}_{2}$ and an analysis of the interband gap function in the $A_{1 g}$ irrep of the $d_{x z}-d_{y z}$ model with $D_{4 h}$ symmetry.

[18] H. Ding, K. Nakayama, P. Richard, S. Souma, T. Sato, T. Takahashi, M. Neupane, Y. M. Xu, Z. H. Pan, A. V. Fedorov, Z. Wang, X. Dai, Z. Fang, G. F. Chen, J. L. Luo, and N. L. Wang, Electronic structure of optimally doped pnictide $\mathrm{Ba}_{0.6} \mathrm{~K}_{0.4} \mathrm{Fe}_{2} \mathrm{As}_{2}$ : A comprehensive angle-resolved photoemission spectroscopy investigation, J. Phys.: Condens. Matter 23, 135701 (2011).

[19] J. R. Schrieffer, Theory of Superconductivity (CRC Press, Boca Raton, 2018).

[20] For example, the direct Coulomb interactions in multiorbital models, including Hund's coupling, are instantaneous and are thus independent of the energy separation between any pair of electrons.

[21] K. V. Samokhin, Exotic interband pairing in multiband superconductors, Phys. Rev. B 101, 214524 (2020).

[22] W. Huang, Y. Zhou, and H. Yao, Exotic Cooper pairing in multiorbital models of $\mathrm{Sr}_{2} \mathrm{RuO}_{4}$, Phys. Rev. B 100, 134506 (2019).

[23] J. Ahn and N. Nagaosa, Theory of optical responses in clean multi-band superconductors, Nat. Commun. 12, 1617 (2021).

[24] J. Ahn and N. Nagaosa, Superconductivity-induced spectral weight transfer due to quantum geometry, Phys. Rev. B 104, L100501 (2021).

[25] H. Molegraaf, C. Presura, D. van der Marel, P. Kes, and M. Li, Superconductivity-induced transfer of in-plane spectral weight in $\mathrm{Bi}_{2} \mathrm{Sr}_{2} \mathrm{CaCu}_{2} \mathrm{O}_{8+\delta}$, Science 295, 2239 (2002). 